\title{
Risk Assessment of Clinical Reactions to Legumes in Peanut-Allergic Children
}

\author{
Louise Bjerremann Jensen, *// Milene Andersen, $\neq$ Per Stahl Skov,//\$ \\ Lars K. Poulsen,// and Carsten Bindslev-Jensen $¥$
}

\begin{abstract}
Peanut-allergic children might be at risk for reactions to other legumes. However, it is not always possible to perform multiple oral food challenges in children. On the basis of patient case history, in vitro diagnostic tests, and eventually food challenges, we aimed at developing an algorithm for risk assessment of possible clinical reactions to other legumes (soybean, lupine, fresh, and blanched green pea). Seventy-five consecutive patients with a positive oral food challenge to peanut were included in the study. All tests were run as part of the routine allergy examination. A high proportion of patients and/or caretakers refused the administered legume oral food challenges. Obtained diagnoses from histamine release did not correlate significantly to the outcome of the algorithm. Interestingly, threshold from peanut challenges did not correlate with the risk assessment.

The algorithm presented in this study can be used when advising peanut-allergic children and their caretakers about what other legumes to avoid in the diet.
\end{abstract}

Key Words: cross-reactivity, histamine release, lupine, oral food challenge, pea, peanut allergy, skin prick test, soy, specific IgE

(WAO Journal 2008;162-167)

$D$ ue to the severity of peanut-allergic reactions, it is important to have specific and standardized methods for allergy diagnosis. The double-blind placebo-controlled food challenge is considered to be the gold standard for food allergy diagnosis. In children below 3 years, it is accepted to perform open food challenges. ${ }^{1}$ The risk associated with an oral food challenge is outweighed by the benefits provided to patients that are shown to be less sensitive (or even tolerant) to the food over time. ${ }^{2,3}$ Moreover, even the most sensitive patients and/or

Received for publication January 7, 2008; accepted July 10, 2008.

From the *Department of Agricultural Sciences, Faculty of Life Sciences, University of Copenhagen, Copenhagen; |Laboratory of Medical Allergology, Allergy Clinic, National University Hospital, Copenhagen; ¥Allergy Centre, Odense University Hospital, Odense; and §RefLab ApS, National University Hospital, Copenhagen, Denmark.

Louise Bjerremann Jensen was funded by a grant from the Faculty of Life Sciences, University of Copenhagen. The study was funded by EU Commission (FAREDAT, QLRT-2001-00301).

Presented at Danish Society of Allergology Annual Meeting, Kolding, Denmark, August 12-13 2005. European Academy of Allergology and Clinical Immunology, Gothenburg, Sweden, June 10-14 2006.

None of the authors have any potential financial conflict of interest related to this manuscript.

Reprints: Carsten Bindslev-Jensen, Allergy Centre, Odense University Hospital, Sdr. Boulevard 29, DK-5000 Odense, Denmark. E-mail: carsten.bindslev-jensen@ouh.fyns@amt.dk.

Copyright (c) 2008 by World Allergy Organization their caretakers benefit from a realistic knowledge of their personal threshold.

Cross-reactivity is observed when preexisting immunoglobulin E (IgE) antibodies recognize similar epitopes on other proteins than the protein they were originally sensitized to, ${ }^{4}$ and several studies on legume cross-reactivity have been published. Magni et $\mathrm{al}^{5}$ investigated the degree of in vitro serologic cross-reactivity between peanut allergens and allergens from soybean and lupine seeds by Western blotting and 2D gel electrophoresis. They found that $\mathrm{IgE}$ antibodies specific for Ara h 3 peanut allergens (an 11S globulin) also recognized soybean and lupine $11 \mathrm{~S}$ globulin basic subunits. ${ }^{5}$ Wensing et $\mathrm{al}^{6}$ reported that patients sensitized to pea exhibited clinically relevant cross-reactivity to peanut where the protein responsible for the cross-reaction was vicilin (Ara h 1 in peanut). ${ }^{6}$

A serological cross-reactivity is, however, not synonymous with allergic reactions in the patients. BernhiselBroadbent and Sampson ${ }^{7}$ and Bernhisel-Broadbent et $\mathrm{al}^{8}$ found in a pediatric population an extensive serologic crossreactivity between legume species that was not reflected clinically. On the one hand, it is argued that related allergens can cause unexpected clinical reactions and, on the other hand, that these structures can mimic sensitization without clinical manifestation, which may be a concern in the diagnosis of patients. $^{9}$

Although oral food challenges would be ideal to establish clinical reactivities to other legumes, it is clearly not feasible to perform 4 to 8 additional food challenges in peanut-allergic children in the daily routine.

Because peanut-allergic children may be at risk for reactions to legumes but cannot always be challenged with all suspected foods, we aimed at developing an algorithm for risk assessment of possible clinical reactions to other legumes (soybean, lupine, fresh, and blanched green pea). On the basis of case history, in vitro diagnostic tests, and, when accepted by the patient, food challenge, the child was classified to a highor a low-risk group. Furthermore, we compared the threshold for peanut challenge and tested the ability of histamine release (HR) as independent diagnostic tools to discriminate between high- and low-risk patients.

\section{MATERIALS AND METHODS}

\section{Patients}

The inclusion criterion for the study was a peanut allergy confirmed by oral food challenge. Seventy-five consecutive patients were included in the study: 46 males $(61 \%)$ and 
29 females (39\%). All patients attended the Allergy Centre at Odense University Hospital, and all tests were run as a part of the routine allergy examination in the period from November 2000 to November 2005.

All patients but 1 were children or adolescents. The age of the patients at the time of the positive oral food challenge was between 7 months and 42 years, the median age being 5 years and 9 months. One patient was not subjected to an oral food challenge with peanut due to a clear-cut case history of peanut allergy but was still included in the study as peanut challenge positive according to the European Academy of Allergology and Clinical Immunology guidelines. ${ }^{1}$

Titrated peanut challenges were performed as described by Taylor et al, ${ }^{10}$ and the content of peanut in the challenge meal inducing subjective and/or objective symptoms was determined. Also, the cumulative dose amount of peanut was recorded. The dose yielding the first mild objective symptoms corresponds to the lowest observed adverse effect level as reported by Taylor et al. ${ }^{11}$ Detailed description of the threshold doses obtained from the peanut challenges will be published elsewhere.

The investigations applied were routine procedures, and therefore the local ethics board stated that formal approval was unnecessary. All testing was only carried out in accordance with the parents and patients wishes.

\section{Legume-Specific Risk Assessment}

After diagnosis of peanut allergy based on the positive challenge outcome, it was investigated whether the patients reacted to other legumes. The patients and/or their caretakers were asked about observed reactions to soybean, lupine, fresh green pea, and blanched green pea, which all belong to the botanical legume family. The answers obtained were grouped in 3 categories: "case history positive or avoids," which included the patients that experienced symptoms upon ingestion and the patients that avoided the food to prevent the induction of symptoms from possible cross-reactions; "case history negative and eats regularly," which included the patients that ate the food without experiencing symptoms; and "case history unknown," which included patients with no known case history. The patients with a positive case history or that avoided the foods were administered a food challenge, but they all refused due to fear of eliciting symptoms during challenge. These patients were categorized as having a high risk of experiencing a reaction after legume consumption. The patients with an unknown case history were subjected to skin

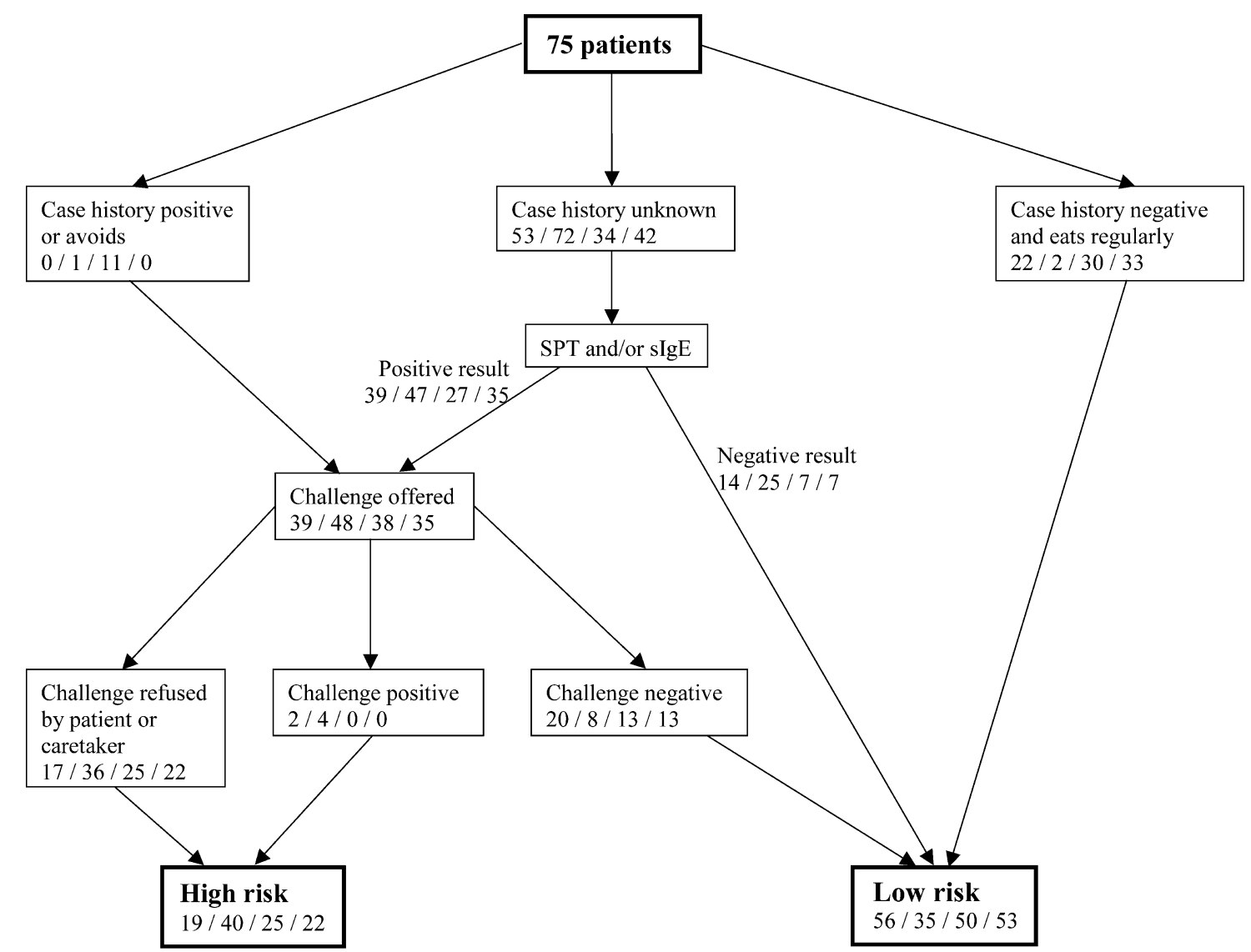

FIGURE 1. Flow sheet of the algorithm. The numbers in each box correspond to the number of patients in the risk assessment of possible reactions to soybean/lupine/fresh green pea/blanched green pea, respectively. Eventually, the patients are divided into high- or low-risk groups. Pea challenges were only performed with fresh green pea. The outcome of the fresh pea challenge was used to classify the patients as either high or low risk for both fresh and blanched green pea. 
TABLE 1. Frequencies of Positive Diagnostic Tests in Peanut-Allergic Patients

\begin{tabular}{llll}
\hline Legume & \multicolumn{1}{c}{ SPT } & HR & sIgE \\
\hline Peanut & $97 \%(68 / 70)$ & $92 \%(45 / 49)$ & $97 \%(69 / 71)$ \\
Soybean & $28 \%(17 / 60)$ & $19 \%(9 / 47)$ & $58 \%(37 / 64)$ \\
Lupine & $45 \%(23 / 51)$ & $80 \%(43 / 54)$ & $47 \%(22 / 47)$ \\
Fresh green pea & $41 \%(17 / 41)$ & $91 \%(39 / 43)$ & $48 \%(23 / 48)$ \\
Blanched green pea & $16 \%(6 / 38)$ & $5 \%(2 / 43)$ & \\
\hline \multicolumn{4}{r}{ Values in parentheses are the number of patients with positive outcome of the tests } \\
divided by the number of patients having the tests performed. \\
\hline
\end{tabular}

prick test (SPT) and/or measurement of specific $\operatorname{IgE}(\mathrm{sIgE})$. If 1 or both results came out positive, the patients were administered a food challenge. If the challenge was refused or the outcome of the challenge was positive, the patients were grouped in the "high-risk" group. A negative challenge outcome grouped the patients in the "low-risk" group. The patients with negative SPTs and sIgE diagnoses were categorized as "low-risk" patients based on the high negative predictive value of SPT and SIgE diagnoses. ${ }^{7,12}$ Finally, the patients that had a negative case history and ate the legume foods regularly were grouped in the "low-risk" group.

The flow sheet in Figure 1 depicts the algorithm by which the patients were classified into 2 groups, reflecting either a high risk or a low risk of possible reaction to the legumes. The outcome of this risk assessment was used in the guidance of the patients and/or caretakers.

\section{Legumes}

Commercially available products of peanut (Arachis hypogaea), soybean (Glycine max), lupine (Lupinus angustifolius), and green pea (Pisum sativum) bought locally were tested in the study because they are common legumes in the diet in Denmark. Both fresh and blanched green peas were tested, where blanched green peas were peas that were briefly blanched in the manufacturing process before they were frozen and sold.

\section{Skin Prick Test}

Skin prick test was performed with peanut, soybean, lupine, fresh green pea, and blanched green pea by the prickprick method. Also, a negative (saline) and positive control (histamine; ALK-Abelló, Hørsholm, Denmark) was included. The criterion for positive result of the test was a wheal with a diameter of $3 \mathrm{~mm}$ or greater, a negative saline control result, and a positive histamine control result.

TABLE 2. Concordances for Pairwise Comparison of the Diagnostic Tests: SPT, HR, and slgE

\begin{tabular}{lccc}
\hline Legume & SPT vs HR, \% & SPT vs IgE, \% & HR vs IgE, \% \\
\hline Peanut & 88 & 97 & 90 \\
Soybean & 80 & 70 & 57 \\
Lupine & 67 & 67 & 57 \\
Fresh green pea & 53 & 63 & 52 \\
Blanched green pea & 79 & 63 & 57 \\
\hline
\end{tabular}

\section{Measurement of slgE}

Serum samples were analyzed for IgE specific for peanut, soybean, lupine, and green pea using the Phadia ImmunoCAPTM system based on a fluoroenzyme immunoassay (Phadia, Uppsala, Sweden). The cutoff for a positive diagnosis was $0.35 \mathrm{kUA} / \mathrm{L}$.

\section{Direct HR}

Patients were tested in basophil HR by direct stimulation of patient basophils. Heparinized blood from the patients was washed with piperazine- $N$ - $N$ '-bis-2-ethanesulfonic acid (PIPES), pH 7.4 (3.02 g/L PIPES, $19.05 \mathrm{~g} / \mathrm{L}$ sodium acetate, $0.49 \mathrm{~g} / \mathrm{L}$ potassium acetate, $1.0 \mathrm{M}$ Tris buffer, $0.088 \mathrm{~g} / \mathrm{L}$ sodium chloride, $0.224 \mathrm{~g} / \mathrm{L}$ magnesium chloride; RefLab ApS, Copenhagen, Denmark). Supernatant was discarded, and cells were resuspended in PIPES with interleukin-3 and stimulated with legumes in 6 different concentrations. The legumes used were peanut, soybean, lupine, fresh green pea, and blanched green pea. Legume extracts were performed as follows: $1 \mathrm{~g}$ of legume in $10 \mathrm{~mL}$ PIPES was grinded in a Stomacher 80 (Seward Medical Limited, London, UK) for 60 seconds at high speed with $10 \mathrm{~mL}$. The sample was centrifuged $(3000 \times g$, 10 minutes, $5^{\circ} \mathrm{C}$ ), and the supernatant was saved as stock solution. The stock solution was diluted 1:10, and this dilution was used as the strongest dilution. Five subsequent 3.5-fold dilutions were prepared. The legume dilutions $(25 \mu \mathrm{l})$ were applied to the glass microfiber plates together with $25 \mu \mathrm{L}$ patient blood sample containing $2 \mathrm{ng} / \mathrm{mL}$ interleukin-3.

The histamine was measured spectrofluorometrically according to the glass microfiber method HR-Test at RefLab ApS described by Stahl Skov et al. ${ }^{13}$ An HR of $10 \mathrm{ng} / \mathrm{mL}$ or greater was considered a positive test result. Positive results were classified in HR classes 1, 2, and 3, where patients with positive reactions to 1 or 2 dilutions were classified as HR class 1 , patients reacting positive to 3 or 4 dilutions were classified as HR class 2 , and patients reacting positive to 5 or all 6 dilutions were classified as HR class 3.14

TABLE 3. Mean SPT, Mean HR Class, and Median slgE for Patients Grouped Into Positive, Negative, or Unknown Case History (Number of Patients in Brackets)

\begin{tabular}{lcccc}
\hline & & $\begin{array}{c}\text { Positive Case } \\
\text { History }\end{array}$ & $\begin{array}{c}\text { Negative Case } \\
\text { History }\end{array}$ & $\begin{array}{c}\text { Unknown } \\
\text { Case History }\end{array}$ \\
\hline Soybean & SPT & & $1(21)$ & $2.5(41)$ \\
& HR & & $0.1(19)$ & $0.5(28)$ \\
& sIgE & & $0.3(21)$ & $0.7(45)$ \\
Lupine & & & & \\
& SPT & $-(0)$ & $4(2)$ & $2.5(54)$ \\
& HR & $-(0)$ & $0(1)$ & $1.3(25)$ \\
Fresh green pea & SIgE & $-(0)$ & $-(0)$ & $0.3(47)$ \\
& & & & \\
& SPT & $2.7(7)$ & $1.5(23)$ & $2.8(30)$ \\
& HR & $1.3(6)$ & $1.0(24)$ & $1.2(25)$ \\
Blanched green pea & SIgE & $1(6)$ & $0.3(20)$ & $0.4(25)$ \\
& & & & \\
& SPT & & $1.2(27)$ & $1.9(32)$ \\
& HR & & $0.2(29)$ & $0.2(26)$ \\
& sIgE & & $0.3(28)$ & $0.9(23)$ \\
\hline
\end{tabular}




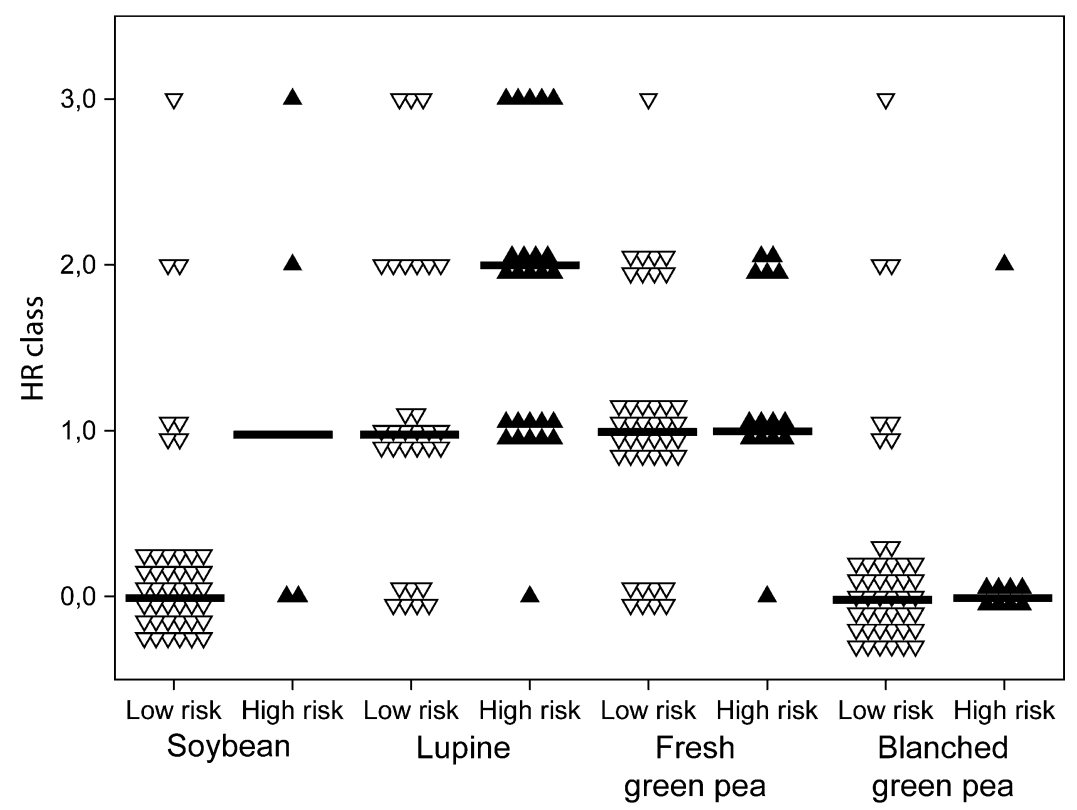

FIGURE 2. Comparison between high- and low-risk classification and HR class for the soybean, lupine, fresh green pea, and blanched green pea. Black lines represent the median.

Histamine release was not a part of the allergy diagnostic workup and was only performed if there was an indication of a reaction. Therefore, HR data were not included in the algorithm.

\section{Statistics}

Specific IgE results were compared with case history outcomes by $t$ test. Obtained HR classes were compared with the classification of patients into high- and low-risk groups by $\chi^{2}$ test. Threshold levels from peanut challenges were compared with the classification of patients into high- and low-risk groups by $t$ test.

\section{RESULTS}

All 75 patients in the study had a clinically confirmed peanut allergy based on the European Academy of Allergology and Clinical Immunology guidelines. ${ }^{1}$

Table 1 shows frequencies of positive results obtained with the 3 different diagnostic tests: SPT, HR, and sIgE. The

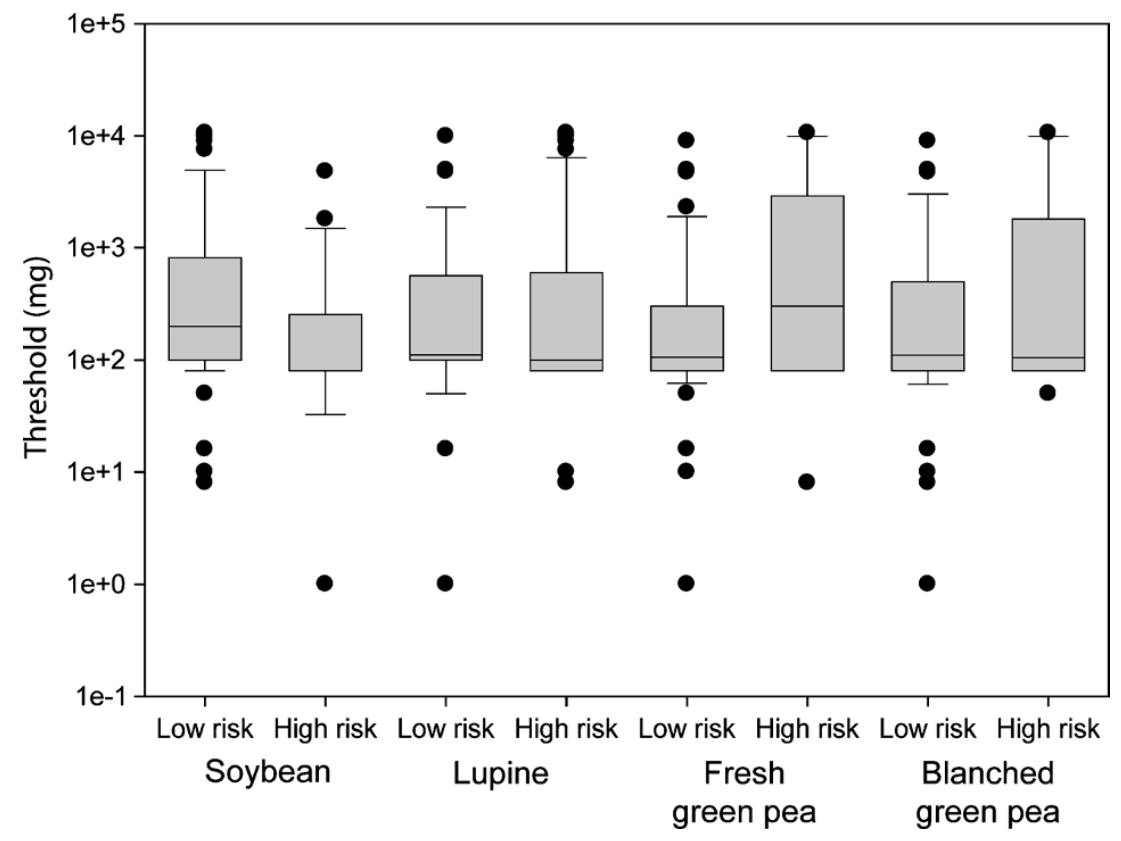

FIGURE 3. Box plot depicting the comparison between high- and low-risk classification and threshold doses of the peanut challenge. 
diagnostic tests SPT and HR produced most positive results for lupine and fresh green pea, followed by soybean and blanched green pea. The measurements of sIgE gave most positive results for soybean followed by pea and lupine.

Pairwise comparisons between the 3 diagnostic tests were performed (Table 2). In 4 of 5 , the concordance was lowest between HR and sIgE.

The flow sheet depicting the developed algorithm for risk assessment of the patient's potential reaction to other legumes is depicted in Figure 1. A high fraction of the patients had an unknown case history to the legumes (71\% for soybean, $96 \%$ for lupine, $45 \%$ for fresh green pea, and $56 \%$ for blanched green pea). The patients in this group with either a positive SPT and/or sIgE were administered an oral food challenge.

A high proportion of patients and/or caretakers refused the oral food challenge (44\% for soybean, $75 \%$ for lupine, $66 \%$ for fresh green pea, and $65 \%$ for blanched green pea). The positive case history or the positive result of the in vitro diagnosis may explain these high proportions of patients refusing challenge.

The fraction of the patients designated to the high-risk group was $25 \%$ for soybean, $53 \%$ for lupine, $33 \%$ for fresh green pea, and $29 \%$ for blanched green pea.

Table 3 depicts the means of SPT and HR, as well as the median of sIgE measurements, where the patients were grouped in positive, negative, and unknown case history. Significant differences $(P<0.05)$ were observed for $\operatorname{sigE}$ results for fresh green pea between patients with positive and negative case history $(P=0.007)$ and for sIgE results for blanched green pea between patients with negative and unknown case history. Generally, the mean for SPT and HR and the median for sIgE were lowest for patients with negative case history $(P=0.047)$.

The outcome of the HR diagnostic test was compared with the classification of patients into high- and low-risk groups. Figure 2 shows the distribution of the HR classes between high- and low-risk groups. There is a tendency for soybean and lupine that HR classes are higher for patients in the high-risk groups; however, this was not significant. Figure 2 indicates that the HR test was too sensitive for fresh green pea.

Peanut HR classes were also compared with the highand low-risk classification. No difference was observed, partly explainable by the fact that 39 of 49 patients were HR class 3 on which the HR analyses was performed (data not shown).

The outcome of the risk assessment was also compared with the threshold of the peanut challenge (Fig. 3). Interestingly, no correlation was observed for any of the legumes.

\section{DISCUSSIONS}

There is increasing evidence that patients with peanut allergy frequently cross-react serologically with other legumes, but only a subpopulation of these patients experience clinical reactions to the legumes. The frequency of clinical cross-reactivity to legumes in peanut-allergic patients is not clear because these patients either avoid legumes, are not aware of the existence of legumes in different foods, or refuse to be orally challenged with these foods.
The aim of the present study was to examine frequency of serological cross-reactivity to soybean, lupine, fresh, and blanched green pea and to compare these results with challenge and case history outcome. This approach was the basis for the development of an algorithm by which it is possible to divide the peanut-allergic patients into a high- and low-risk group reflecting the precautions the patients must take in the diet.

Histamine release as an independent diagnostic test could not predict the outcome of the algorithm giving the highand low-risk classification. The comparison of SPT and sIgE with the algorithm outcome was not performed because SPT and SIgE were a part of the algorithm.

We expected the most sensitive peanut-allergic patients to also experience more clinically relevant cross-reactivity to the other legumes. Interestingly, the high- and low-risk classification was not dependent of the threshold dose of the peanut challenge.

Twenty-eight percent of the patients had a positive SPT to soybean, whereas for only 2 of 44 patients $(5 \%$; the 22 with a negative case history were included as challenge negative), the allergy was confirmed clinically by challenge. These numbers are in agreement with Bock and Atkins ${ }^{15}$ who investigated 32 clinically confirmed peanut-allergic patients. They found that 10 patients $(31 \%)$ had a positive soybean SPT, whereas only 1 patient $(3 \%)$ had a clinical reaction after ingestion of soybean.

Case history reports from the patients and/or caretakers reflect that lupine is a new and unknown source of allergens important for peanut-allergic individuals. Only 3 patients were aware of having ingested or reacted to lupine, and of these, 1 had a positive case history. These data indicate that the awareness of lupine as a food allergen in Denmark is still sparse. A search for the combination of "peanut," "allergy," and "lupine" at PubMed gave only 13 hits, where the first is published in 1994 and is a report on reactions to lupine added to pasta. ${ }^{16}$ It is important to emphasize that the risk of coallergy to lupine for peanut-sensitized subjects might be higher than for other legumes, but more knowledge needs to be gained on this matter. Moneret-Vautrin et al ${ }^{17}$ investigated 24 peanut-allergic patients, and they found that $44 \%$ had a positive SPT to lupine, which is in agreement with our data where $45 \%$ had a positive lupine SPT. These authors also found that the risk of crossed peanut-lupine allergy was higher compared with cross-allergy to other legumes. This risk combined with the fact that lupine is added frequently to foods as an excellent protein source is making lupine an allergen of interest for future research.

Positive SPT frequencies were $41 \%$ and $16 \%$ for fresh and blanched green pea, respectively. Bernhisel-Broadbent and Sampson ${ }^{7}$ investigated 69 patients with 1 or more positive SPTs to legumes in 1989 and found that $26 \%$ of pea SPTs were positive (it is not stated whether fresh or blanched green pea was used).

Eleven patients reported symptoms upon ingestion of fresh green pea, whereas no patients observed symptoms after ingestion of blanched green pea. The degradation of pea allergens by heat treatment can explain this. Peanut and lupine has been reported to be stable to heat treatment. ${ }^{18-20}$ This 
stability can explain that clinically relevant reactions are seen to peanut and lupine.

Many patients were not challenged in the study but were still assigned to the high-risk group. Although no scientific reason is given for this classification, it is advisable from a practical point of view to consider these patients as high-risk patients until the opposite has been proven.

This study is the first to standardize the process of diagnosing peanut-allergic children with regard to possible cross-reactions to other foods. By using the algorithm presented in this study, it is possible to advise peanut-allergic children and caretakers on which other legumes to avoid in their diet.

\section{ACKNOWLEDGMENTS}

The authors thank Lise Bo at RefLab ApS and Ulla Johannessen at the Allergy Centre, Odense University Hospital.

\section{REFERENCES}

1. Bindslev-Jensen C, Ballmer-Weber BK, Bengtsson U, Blanco C, Ebner C, Hourihane J, et al. Standardization of food challenges in patients with immediate reactions to foods - position paper from the European Academy of Allergology and Clinical Immunology. Allergy. 2004;59(7):690-697.

2. Skolnick HS, Conover-Walker MK, Koerner CB, Sampson HA, Burks W, Wood RA. The natural history of peanut allergy. J Allergy Clin Immunol. 2001;107(2):367-374.

3. Perry TT, Matsui EC, Conover-Walker MK, Wood RA. Risk of oral food challenges. J Allergy Clin Immunol. 2004;114(5):1164-1168.

4. Aalberse RC. Structural biology of allergens. J Allergy Clin Immunol. 2000;106(2):228-238.

5. Magni C, Ballabio C, Restani P, Sironi E, Scarafoni A, Poiesi C, et al. Two-dimensional electrophoresis and Western-blotting analyses with anti Ara h 3 basic subunit IgG evidence the cross-reacting polypeptides of Arachis hypogaea, Glycine max, and Lupinus albus seed proteomes. $J$ Agric Food Chem. 2005;53(6):2275-2281.

6. Wensing M, Knulst AC, Piersma S, O'Kane F, Knol EF, Koppelman SJ. Patients with anaphylaxis to pea can have peanut allergy caused by cross-reactive IgE to vicilin (Ara h 1). J Allergy Clin Immunol. 2003; 111(2):420-424.

7. Bernhisel-Broadbent J, Sampson HA. Cross-allergenicity in the legume botanical family in children with food hypersensitivity. $J$ Allergy Clin Immunol. 1989;83(2 Pt 1):435-440.

8. Bernhisel-Broadbent J, Taylor S, Sampson HA. Cross-allergenicity in the legume botanical family in children with food hypersensitivity: II. Laboratory correlates. J Allergy Clin Immunol. 1989;84(5 Pt 1): 701-709.

9. Sicherer SH. Clinical implications of cross-reactive food allergens. $J$ Allergy Clin Immunol. 2001;108(6):881-890.

10. Taylor SL, Hefle SL, Bindslev-Jensen C, Atkins FM, Andre C, Bruijnzeel-Koomen $\mathrm{C}$, et al. A consensus protocol for the determination of the threshold doses for allergenic foods: how much is too much? Clin Exp Allergy. 2004;34(5):689-695.

11. Taylor SL, Hefle SL, Bindslev-Jensen C, Bock SA, Burks AW, Christie L, et al. Factors affecting the determination of threshold doses for allergenic foods: how much is too much? J Allergy Clin Immunol. 2002; 109(1):24-30.

12. Sampson HA, Albergo R. Comparison of results of skin tests, RAST, and double-blind, placebo-controlled food challenges in children with atopic dermatitis. J Allergy Clin Immunol. 1984;74(1):26-33.

13. Stahl SP, Norn S, Weeke B. A new method for detecting histamine release. Agents Actions. 1984;14(3-4):414-416.

14. Nolte H, Storm K, Schiotz PO. Diagnostic value of a glass fibre-based histamine analysis for allergy testing in children. Allergy. 1990;45(3):213-223.

15. Bock SA, Atkins FM. The natural history of peanut allergy. J Allergy Clin Immunol. 1989;83(5):900-904.

16. Hefle SL, Lemanske RF Jr, Bush RK. Adverse reaction to lupine-fortified pasta. J Allergy Clin Immunol. 1994;94(2 Pt 1):167-172.

17. Moneret-Vautrin DA, Guerin L, Kanny G, Flabbee J, Fremont S, Morisset M. Cross-allergenicity of peanut and lupine: the risk of lupine allergy in patients allergic to peanuts. J Allergy Clin Immunol. 1999; 104(4 Pt 1):883-888.

18. Astwood JD, Leach JN, Fuchs RL. Stability of food allergens to digestion in vitro. Nat Biotechnol. 1996;14(10):1269-1273.

19. Alvarez-Alvarez J, Guillamon E, Crespo JF, Cuadrado C, Burbano C, Rodríguez J, et al. Effects of extrusion, boiling, autoclaving, and microwave heating on lupine allergenicity. J Agric Food Chem. 2005;53(4):1294-1298.

20. Maleki SJ, Chung SY, Champagne ET, Raufman JP. The effects of roasting on the allergenic properties of peanut proteins. J Allergy Clin Immunol. 2000;106(4):763-768. 